\title{
Subtemporal Approach
}

\author{
Yong Bae Kim and Kyu Sung Lee
}

\subsection{Introduction}

Historically, the first form of subtemporal approach had been described for the treatment of trigeminal neuralgia early in the twentieth century. Temporal craniotomies had offered surgical corridor to the various lesions situated in middle cranial fossa. By the 1960s, subtemporal approach entered in flourishing period by Drake's pioneering work of surgical treatment for more than 1700 basilar and posterior circulation aneurysms. Subsequently, modifications of subtemporal route, such as subtemporal key hole approach, extended exposure with zygomatic resection, or removal of petrous apex, were developed to reach peri-mesencephalic and mid-clival regions.

Here, in this chapter, the subtemporal approach will be illustrated mainly focusing on Drake's original description, while modifications for extended exposure are included for relevant occasions.

\footnotetext{
Y. B. Kim · K. S. Lee $(\bowtie)$

Department of Neurosurgery,

Yonsei University College of Medicine,

Gangnam Severance Hospital, Seoul, South Korea

e-mail: ybkim69@yuhs.ac;

KYUSUNG@yuhs.ac; kyusung@yumc.yonsei.ac.kr
}

\subsection{Steps of the Approach}

After induction of general anesthesia, a lumbar drain is inserted to achieve adequate intraoperative brain relaxation. Albeit Drake and some others prefer to place patient in lateral decubitus position, supine position with the ipsilateral shoulder elevated is usually satisfactory for routine practice. Mostly right-sided (non-dominantsided) approach is selected unless the location and/or direction of the lesion, left third nerve palsy, compromised left vision, or right hemiparesis mandate left-sided approach.

The head is rotated to the contralateral side, aligning anterior-posterior axis parallel with the floor. The vertex is slightly tilted down toward the floor for efficient intraoperative viewing trajectory as the base of the middle cranial fossa inclines upward steeply. The aim of subtemporal approach is to operate as close to the base of middle cranial fossa of the skull as possible. Therefore, the skin incision should be designed to expose temporal squama at least to the extent of root of the zygomatic arch. Usually, an inverted U-shaped skin incision is made, beginning at the level of the root of the zygomatic arch, within $1-2 \mathrm{~cm}$ anterior to the tragus. The incision is curved anteriorly and upwardly and then backward over the pinna around the ear. Alternately, a linear skin incision originating from the inferior rim of the zygomatic arch, approximately one finger's width, anterior to the external auditory 


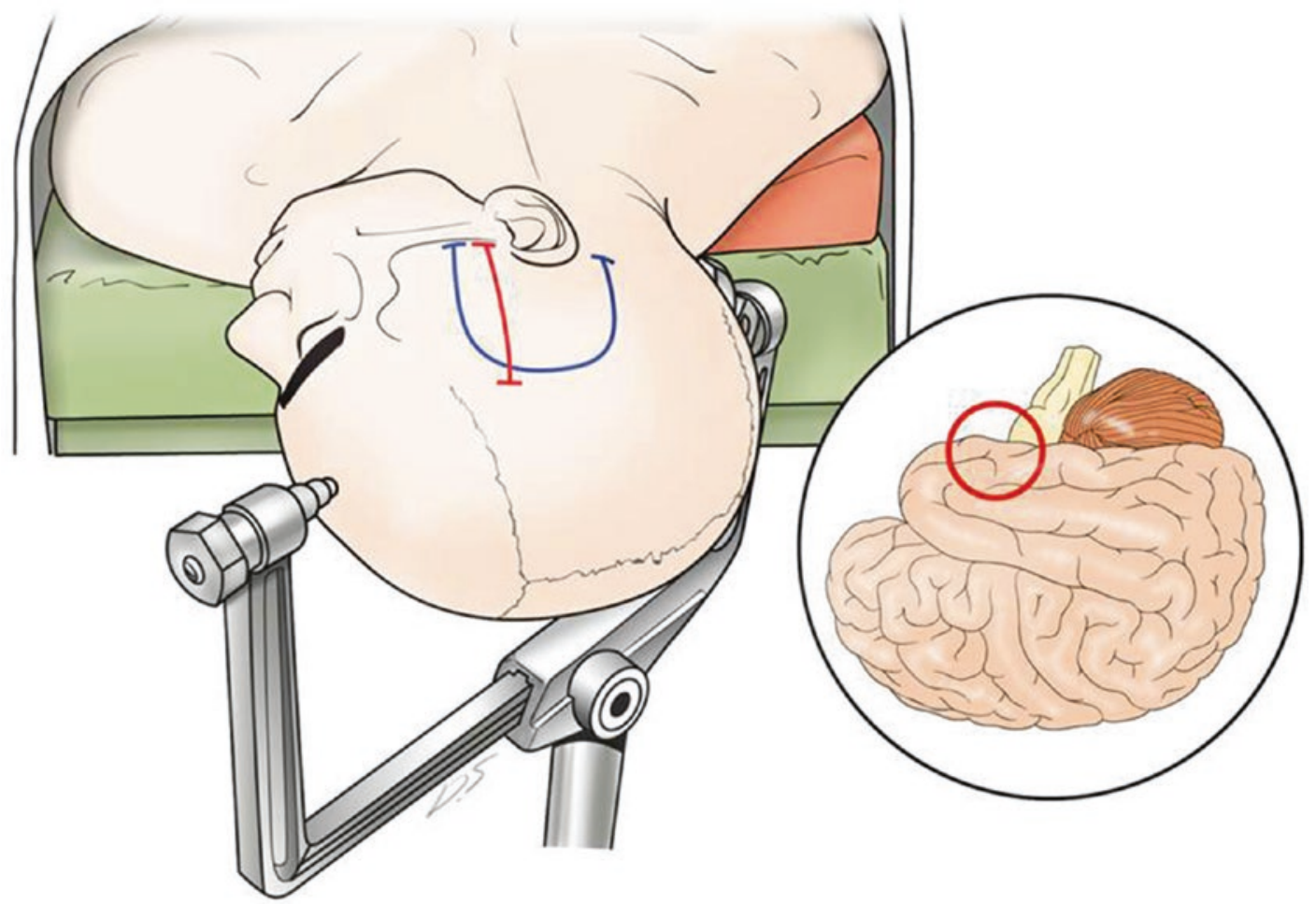

Fig. 6.1 The patient is usually positioned in supine with ipsilateral shoulder elevated. The head is rotated to the contralateral side and slightly tilted down toward the floor.

canal can be adopted for minimized temporal bone flap (Fig. 6.1).

The skin flap is then reflected inferiorly, and the temporalis muscle is divided and reflected as well, exposing the zygomatic process of the temporal bone and the root of the zygomatic arch. A 4-5 cm-sized craniotomy at exposed temporal squama is performed, placing inferior border of the bone flap as lower as possible. If mastoid air cells are inadvertently opened, careful closure by bone waxing or temporal muscle flap should be completed. It is critical to drill down the inferior overhanging edge of the temporal bone to flush with the base of the middle cranial fossa.

The dura is opened and reflected with several tack-up sutures. At this point, the lumbar drain is opened until sufficient brain relaxation is gained. Gentle retraction of the temporal lobe should progress in stepwise manner as the floor of the middle cranial fossa shapes convex. Small bridging veins from undersurface of the temporal lobe to the tent can be divided when necessary.
An inverted U-shaped incision or linear incision can be used to expose the temporal squama just above the zygomatic tubercle

However, care must be taken never to violate the vein of Labbe, which is usually running just beyond the posterior border of the temporal craniotomy. As the temporal lobe is being retracted further, the free edge of the tentorial incisura and the arachnoid sheath of the ambient cistern become visualized. Opening of the ambient cistern will provide further cerebrospinal fluid drainage and clear view of cisternal contents. The trochlear nerve can be identified usually underneath of the tentorium, when the free edge is lifted up only few millimeters. More anteriorly, the interpeduncular cistern comes into view by elevating the uncus. A thick arachnoid membrane, so-called Liliequist's membrane, is extending from the anterior edge of the mammillary bodies to the superior border of the dorsum sellae, separating interpeduncular cistern from the carotid and chiasmatic cisterns. Laterally, this membrane is perforated by the posterior communicating artery and the oculomotor nerve. A vertical incision at the tentorium just behind the entry point of the trochlear nerve will 


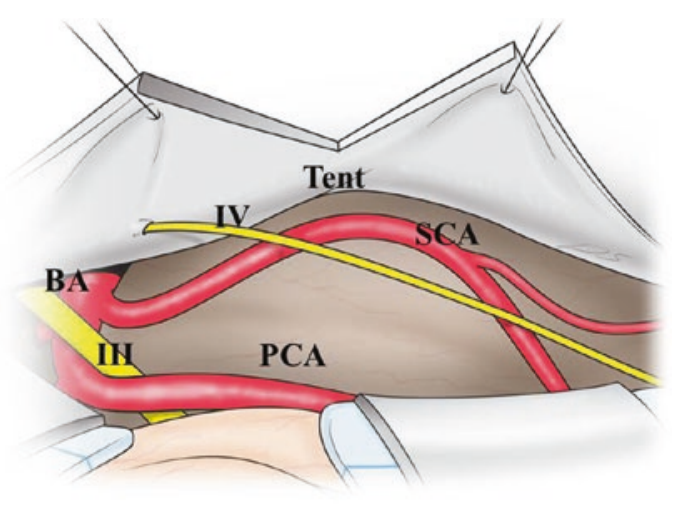

Fig. 6.2 After the temporal lobe is gently retracted, the free edge of the tentorial incisura and the arachnoid sheath of the ambient cistern become visualized. The trochlear is running usually underneath of the tentorium. A vertical incision at the tentorium right posterior to the entry point of the trochlear nerve is made, and the incised leaflets of the tentorium are raised up bilaterally using stay sutures

provide wider opening of the ambient cistern. The incised leaflets of the tentorium are raised up bilaterally using stay sutures. At this stage, the ambient segment of the superior cerebellar artery (SCA) is firstly seen on the anterolateral side of the pontomesencephalic junction. Anteriorly, the oculomotor nerve arises from the medial surface of the cerebral peduncle and traverses between the posterior cerebral artery (PCA) and the SCA (Fig. 6.2). After opening the Liliequist's membrane, the bifurcation of the basilar artery (BA) is found within interpeduncular cistern (Fig. 6.4). The PCA is joined by the posterior communicating artery at the lateral margin of the interpeduncular cistern and passes over the oculomotor nerve along crural and ambient cisterns. The direct perforators to the brain stem and thalamus usually arise from the superior surface of the PCA; therefore, it should be safer to follow the PCA along the inferior surface medially to the basilar apex. If the posterior communicating artery is hypoplastic, and obscuring surgical view around the basilar apex, then the artery may be divided only when perforators are not compromised. The origin of SCA is usually located inferior to the trochlear nerve. When temporary clip of the BA trunk is necessary, a few millimeters segment of the BA below the origin of the SCA is ideal because there is seldom existence of the BA perforators.

\subsection{Indication of the Approach}

For basilar bifurcation aneurysms, the subtemporal approach provides a direct avenue to interpeduncular fossa. However, the relative location of the bifurcation from the posterior clinoid process (PCP) is an important issue. When the bifurcation lies approximately at the level of the PCP, the surgical approach can be straightforward. A very high-positioned bifurcation requires looking-up viewing trajectory; therefore, resection of the zygomatic arch and further retraction of the temporal lobe are mandated. In such case, frontotemporal trans-Sylvian approach could be preferred to the subtemporal approach. A very low-positioned bifurcation may be significantly hampered by narrow corridor even with the tentorial incision, and the dome of the aneurysm often obscures surgeon's path to the neck. The direction of the dome of the aneurysm should also be considered when selecting surgical approach. Placing of the clips becomes straightforward for BA aneurysms projecting anteriorly, as the dome is exposed directly into view while the neck of the aneurysm tends to be free of the PCA and its perforators. On the contrary, posterior-projecting aneurysms may pose difficulties in direct clipping. The dome can be buried high up in the interpeduncular fossa, and numerous perforators from basilar terminus are usually adhered with the back of the aneurysm. It is critical to separate those perforators form the site where the final clip is to be deployed. The neck of the aneurysm is often hidden underneath the ipsilateral oculomotor nerve and the PCA. Such configurations may possess blind spots around the neck of the aneurysm, resulting in incomplete or mispositioned final clip.

For aneurysms arisen at the origin of the SCA, the subtemporal approach provides excellent visualization of the neck and fundus of the aneurysms. They are usually projecting laterally toward the approach side, and no perforators emanate from the BA segment between the PCA and SCA, making clipping simple and straightforward. Only concerns should be given not to compromise or kink the BA and the SCA orifice.

The aneurysms arisen at the P1 (from the origin of the PCA to the junction with the posterior com- 
municating artery) and P2 (within peduncular and ambient cisterns) segment of the PCA can best be dealt with subtemporal approach. One may require resecting some parts of the parahippocampal gyri when the aneurysm is hidden under them.

Because the subtemporal approach exposes mesial temporal lobe, and the lateral aspect of mesencephalon, vascular malformations such as the arteriovenous malformations and cavernous

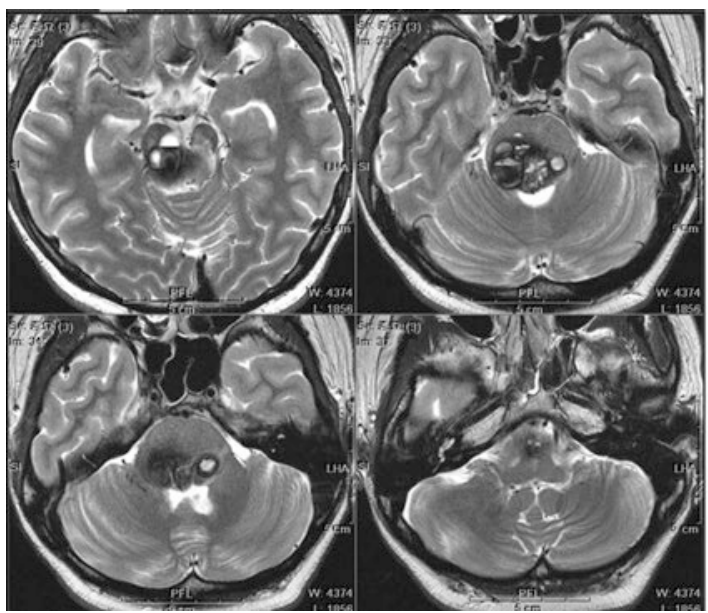

Fig. 6.3 Axial T2-weighted (left) and gadoliniumenhanced T1-weighted (right) magnetic resonance images reveal cavernous malformation residing in upper pons. Note fluid-fluid layer inside the lesion, implying previous episodes of intralesional rupture. Dorsal surface of the

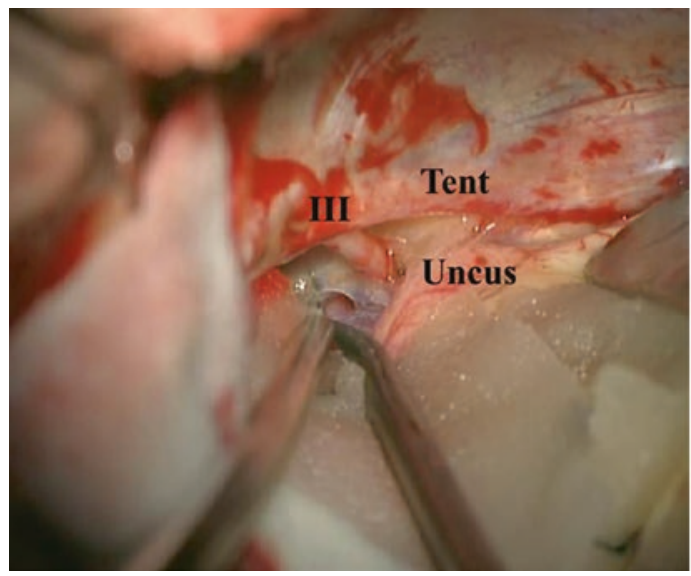

Fig. 6.4 For extended subtemporal exposure, the patient is placed in lateral decubitus position. A curvilinear incision is made extending from the inferior border of the root of the zygomatic arch, curving anteriorly to the midline behind the hairline. By using interfascial technique to avoid facial nerve injury, the zygomatic arch is exposed and resected. After gentle retraction of the temporal lobe, malformations situated around these structures are good candidates for the subtemporal approach. Cavernous malformations or other vascular lesions developed at the level of upper pons can be removed when the subtemporal approach is combined with removal of petrous apex (extended subtemporal exposure, or anterior transpetrosal approach) (Figs. 6.3, 6.4, 6.5, 6.6, 6.7, 6.8, and 6.9). Open surgical treatment for the

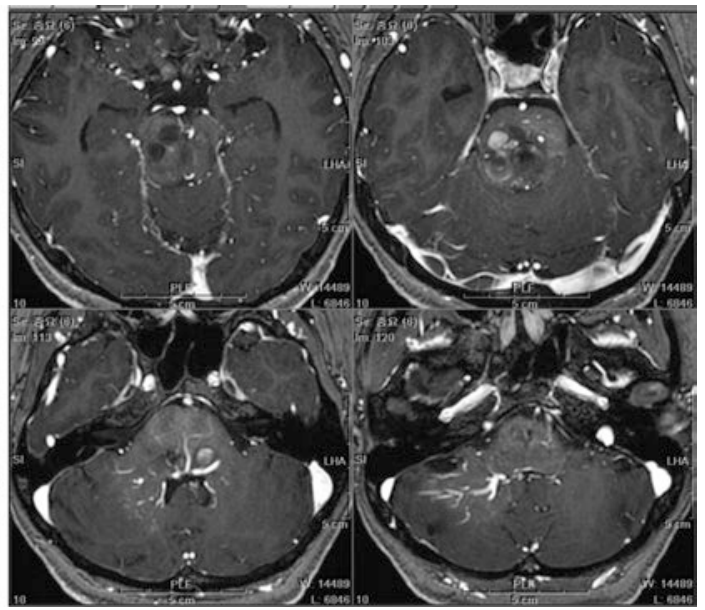

lesion is engulfed by network of the venous malformation; therefore approaches from the midline of the posterior fossa are impossible. Instead, extended subtemporal transtentorial approach is selected to approach from the right posterolateral aspect of the brain stem

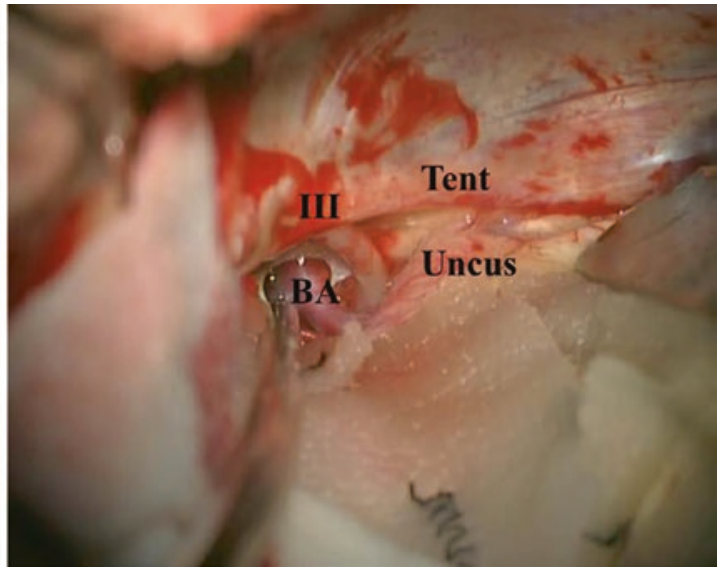

the free edge of the tentorium becomes visualized. The uncus is the landmark to identify the oculomotor nerve (III). Note that the thick arachnoid membrane over the interpeduncular fossa (Liliequist's membrane) is adherent laterally to the oculomotor nerve (left). After the membrane is opened, the terminus of the basilar artery is then exposed (right) 

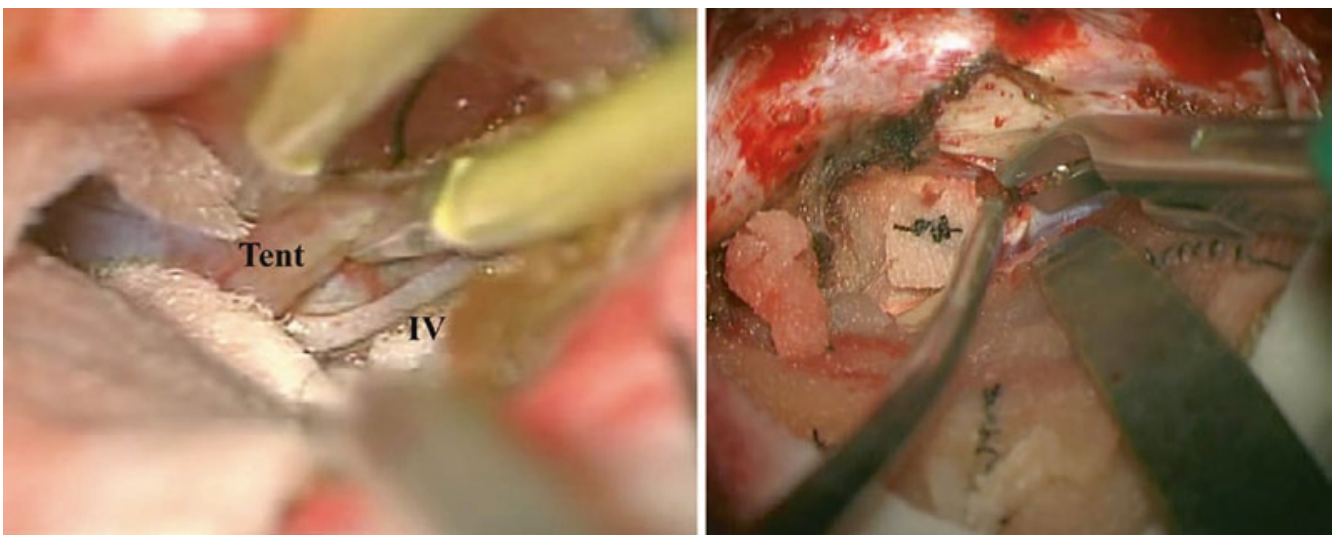

Fig. 6.5 Posteriorly to the oculomotor nerve, the crural and ambient cistern is opened. The tentorium is incised posterior to the entry point of the trochlear nerve to gain wider surgical window (left). The extension of the tento- rial incision is performed toward the superior petrosal sinus to remove dural cover at the area of the petrous apex. The superior petrosal sinus is ligated using hemoclip (right)
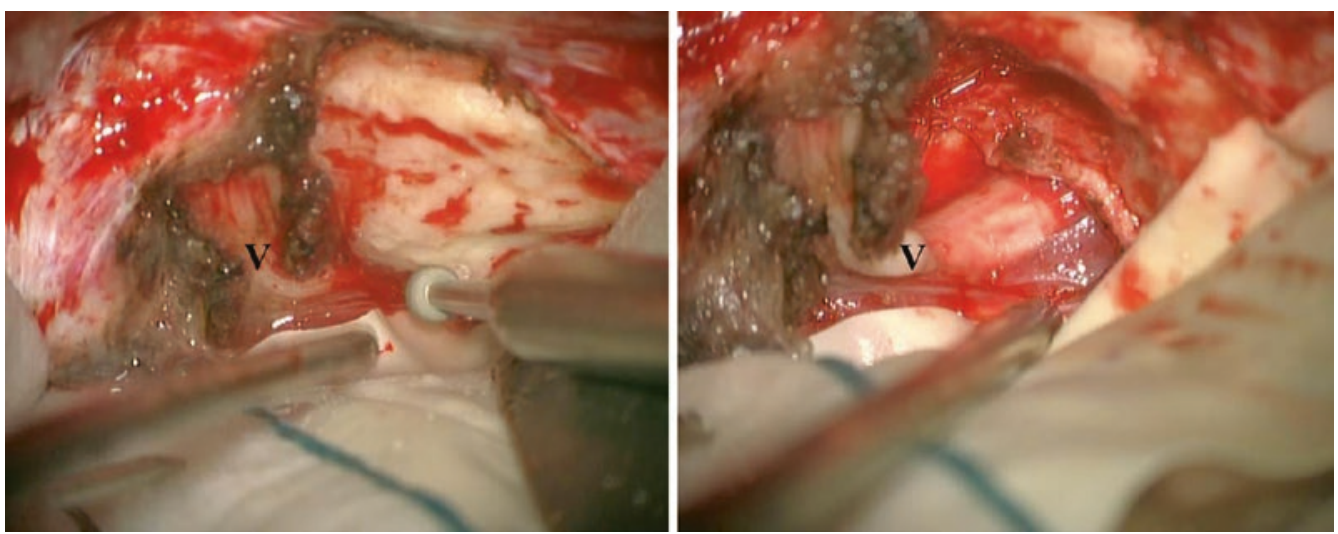

Fig. 6.6 The dura over the Meckel cave and the petrous apex is uncovered (left). With gentle mobilization and protection of the trigeminal nerve, the petrous apex is removed using diamond drill (right)

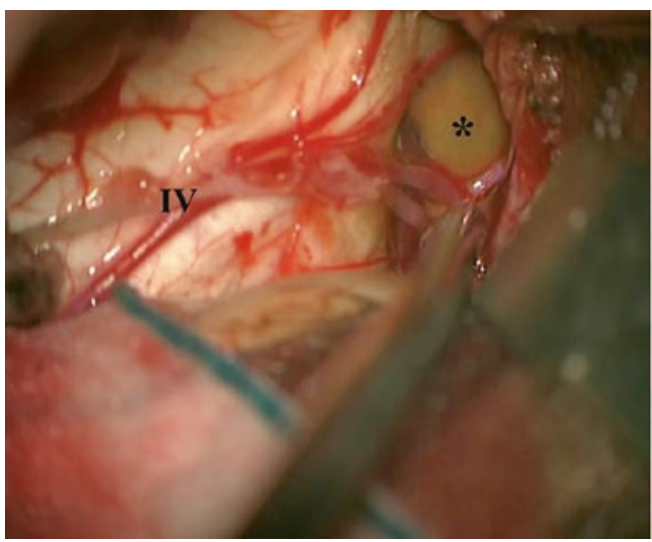

Fig. 6.7 By removal of the petrous apex, upper half of the retroclival region is exposed. Note the yellowish discoloration on the surface of the pons (left, asterisk).

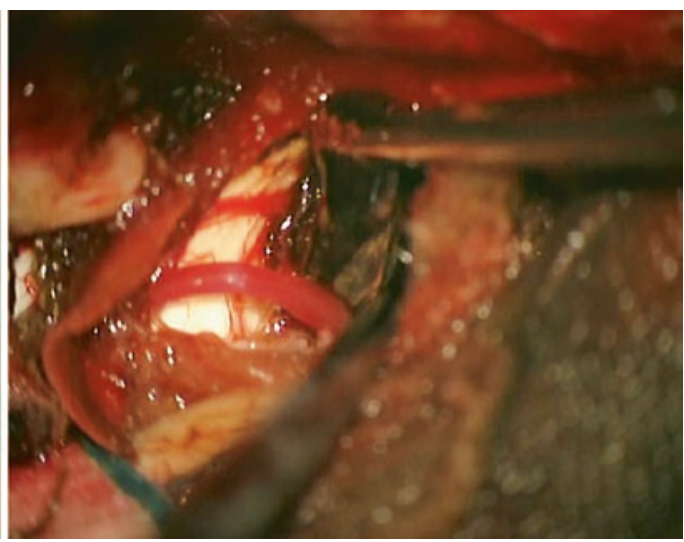

A small incision at the discolored surface of the pons is made, and the cavernous malformation is being removed (right) 


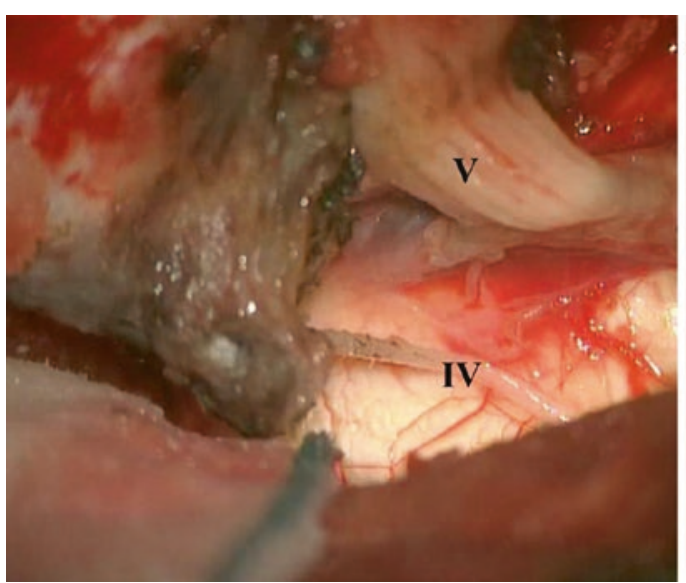

Fig. 6.8 The overall extent of intraoperative view from extended subtemporal transtentorial approach utilizing resection of the zygomatic arch and the petrous apex. The

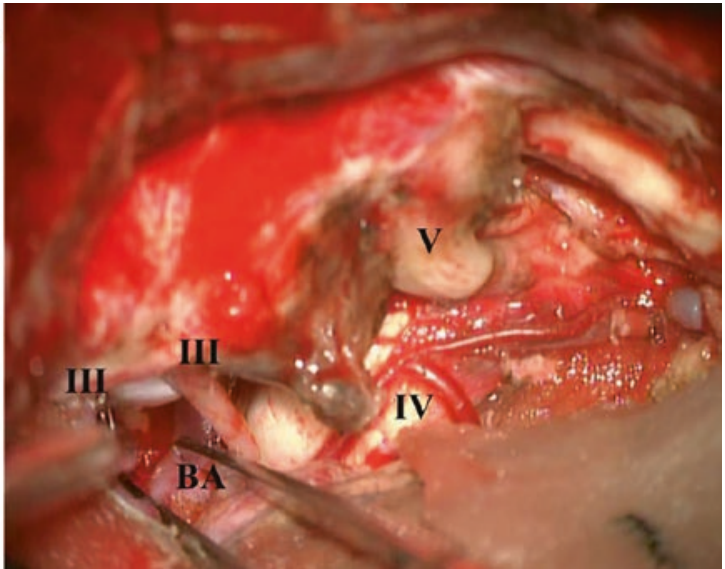

retroclival and anterolateral mesencephalo-pontine area are fully exposed. Note the bilateral oculomotor nerves, the trochlear nerve, the trigeminal nerve, and the BA (right)
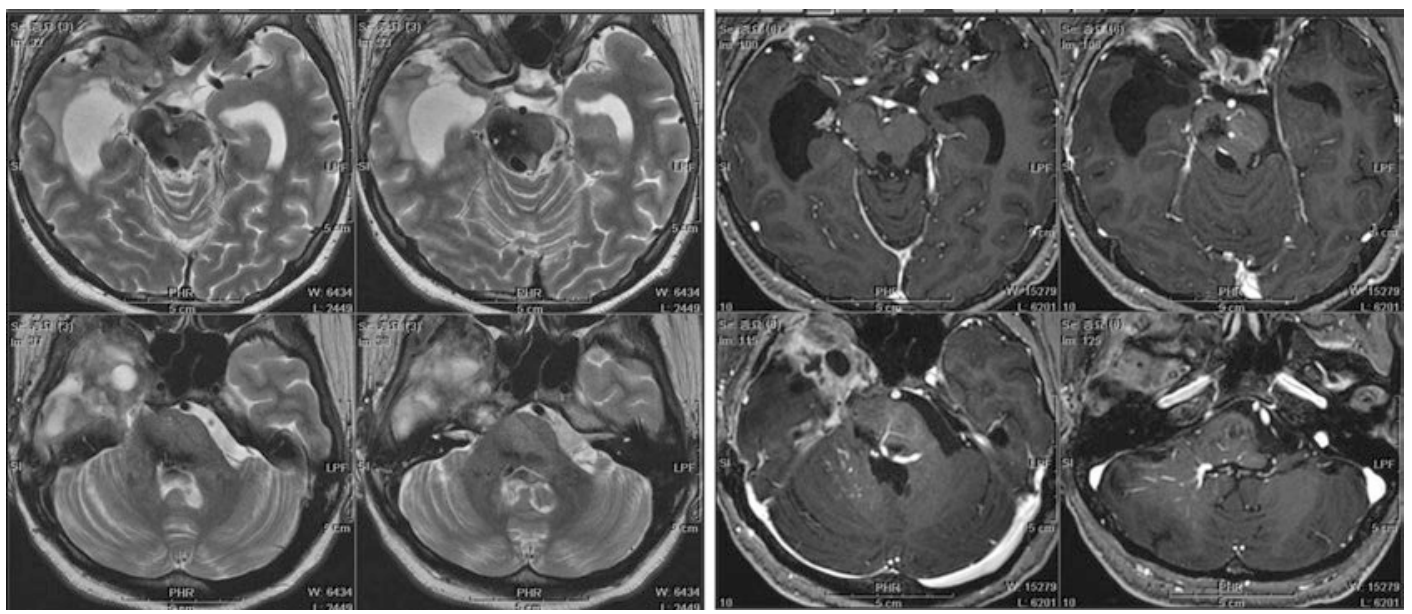

Fig. 6.9 Axial T2-weighted (left) and gadolinium-enhanced T1-weighted (right) magnetic resonance images reveal complete removal of the cavernous malformation. The venous channels at the dorsum of the lesion are well preserved

dural arteriovenous fistulae in the tentorial incisura can also be considered in suitable condition via subtemporal approach.

\subsection{Limitation, Complication, and How to Avoid}

Although the subtemporal approach may provide relatively straightforward exposure of anatomy and an adequate visualization of the terminal basilar complex, the narrow working window of lateral surgical trajectories often permit only limited maneuverability. Therefore, reflecting the tentorial edge is the key step to increase working space around the cerebral peduncle. When making incision at the tentorium, the entrance of the trochlear nerve to its dural canal should be identified first to avoid the trochlear nerve injury.

Nevertheless, the essential part of this approach is assured only by retracting temporal lobe in certain degree. The injury to the temporal 
lobe by pressuring retraction must be one of the most disadvantageous consequences in this approach. Adequate drainage of cerebrospinal fluid is, therefore, of prime importance. When the draining vein is compromised, retraction of the temporal lobe will be more easily prone to postoperative venous infarction. Uncal retraction may cause tethering injury to the oculomotor nerve. Ordinarily, it is advocated to leave the oculomotor nerve attached and ensheathed within thick arachnoid without manipulation to avoid postoperative palsies. In selected cases, it may be necessary to dissect the oculomotor nerve and work both under and above the nerve. Once the integrity of the oculomotor nerve is well preserved, postoperative palsies should be transient. Due to the relatively lateral to medial direction of line of sight, the contralateral PCA and perforating vessels are often difficult to identify so that retraction or indentation of the aneurysm itself may be necessary to visualize the hind aspect of the aneurysm. Selecting appropriate length of clips is also important not to blindly bite the ves- sels and nerves embedded in deep opposite side. Fenestrated clips are very useful for passing the ipsilateral PCA through the aperture.

\section{Bibliography}

1. Drake CG. Surgical treatment of ruptured aneurysms of the basilar artery. Experience with 14 cases. J Neurosurg. 1965;23(5):457-73.

2. Yasargil MG, Antic J, Laciga R, Jain KK, Hodosh RM, Smith RD. Microsurgical pterional approach to aneurysms of the basilar bifurcation. Surg Neurol. 1976;6(2):83-91.

3. Kawase T, Shiobara R, Toya S. Anterior transpetrosaltranstentorial approach for sphenopetroclival meningiomas: surgical methods and results in 10 patients. Neurosurgery. 1991;28:869-76.

4. Hernesniemi J, Ishii K, Niemelä M, Kivipelto L, Fujiki M, Shen H. Subtemporal approach to basilar bifurcation aneurysms: advanced technique and clinical experience. Acta Neurochir Suppl. 2005;94:31-8.

5. McLaughlin N, Martin NA. Extended subtemporal transtentorial approach to the anterior incisural space and upper clival region: experience with posterior circulation aneurysms. Neurosurgery. 2014;10(Suppl 1):15-24.

Open Access This chapter is licensed under the terms of the Creative Commons Attribution 4.0 International License (http://creativecommons.org/licenses/by/4.0/), which permits use, sharing, adaptation, distribution and reproduction in any medium or format, as long as you give appropriate credit to the original author(s) and the source, provide a link to the Creative Commons license and indicate if changes were made.

The images or other third party material in this chapter are included in the chapter's Creative Commons license, unless indicated otherwise in a credit line to the material. If material is not included in the chapter's Creative Commons license and your intended use is not permitted by statutory regulation or exceeds the permitted use, you will need to obtain permission directly from the copyright holder. 